\title{
INVENTÁRIO FLORÍSTICO QUALI-QUANTITATIVO DAS VIAS PÚBICAS DE ITANHAÉM -SP
}

\author{
QUALITATIVE AND QUANTITATIVE FLORISTIC INVENTORY OF STREET TREES IN \\ ITANHAÉM-SP
}

\author{
Tamara Ribeiro Botelho de Carvalho Maria ${ }^{1}$, Daniela Biondi², Rogério Brobowski ${ }^{3}$
}

\begin{abstract}
RESUMO
O objetivo desta pesquisa foi realizar um inventário quali-quantitativo da arborização de ruas de Itanhaém, São Paulo. O município está inserido sob o Bioma Mata Atlântica, apresenta uma área urbanizada de 40,8 ha, e é subdividido em 15 regionais administrativas. Para a avaliação quantitativa foram percorridas as vias urbanizadas do município, contabilizando a quilometragem de vias urbanizadas e o número de árvores. Para o inventário qualitativo, os indivíduos foram avaliados quanto à localização, espécie, condição estrutural e fitossanitária, condição de raiz, altura total, altura de bifurcação, área de canteiro, DAP e necessidades de manejo. Foram encontrados 18.128 indivíduos de porte arbóreo em área urbanizada do munícipio, com Terminalia catappa L. (17,47\%), Ficus benjamina (16,76\%) como as espécies de maior frequência. A maioria dos indivíduos (64\%) estão entre 0 e 6 metros de altura, com DAP médio de $16,8 \mathrm{~cm}$, o que indica expressiva ocorrência de indivíduos arbóreos jovens e ou de pequeno a médio porte. A maioria dos indivíduos apresentou boas condições estruturais e fitossanitárias (54\%), raízes profundas $(64 \%)$ e sem necessitar de práticas de manejo $(49,8 \%)$, entretanto a poda leve foi o manejo mais indicado (27\%).
\end{abstract}

Palavras-chave: Floresta urbana; Arborização de ruas; Amostragem; Composição florística.

\section{ABSTRACT}

The aim of this study was to conduct a qualitative and quantitative inventory of the street trees of Itanhaém, São Paulo. The city is inserted in the Atlantic Forest biome, it has a urbanized area of 40.8 ha and it is divided into 15 administrative regionals. For the quantitative evaluation were covered the municipality urbanized pathways, counting the mileage of urban roads and the number of trees. For the qualitative inventory, subjects were evaluated for location, species, structural and phytosanitary condition, root condition, total height, fork height, site area, DBH and management needs. There were found 18,128 tree-sized individuals in the urban area of the municipality, with Terminalia catappa L. (17.47\%) and Ficus benjamina (16.76\%) as the most frequent species. Most individuals (64\%) are between 0 and 6 meters high, with an average of $16.8 \mathrm{~cm}$ of $\mathrm{DBH}$, which indicates significant occurrence of young individuals or small to medium sized trees. Most individuals had good structural and plant health (54\%), deep roots $(64 \%)$ and no needs for management practices (49.8\%), though the light pruning is the most suitable management (27\%).

Keywords: Urban forest; Street afforestation; Sampling; Floristic composition.

\footnotetext{
Recebido em 11.07.2016 e aceito em 23.09.2016

1 Engenheira Florestal, Mestranda do Programa de pós-graduação em Engenharia Florestal da Universidade Federal do Paraná. Curitiba/PR. E-mail: trbotelhomaria@gmail.com

2 Engenheira Florestal, Prof ${ }^{a} \mathrm{Dr}^{\mathrm{a}}$ da Universidade Federal do Paraná. Curitiba/PR. E-mail: dbiondi@ufpr.br

3 Engenheiro Florestal, Profo ${ }^{\circ} r^{\circ}$ da Universidade Estadual do Centro-Oeste. Irati/PR. E-mail: bobrowski_roger@yahoo.com.br
} 


\section{INTRODUÇÃO}

A atual estrutura da maioria das cidades brasileiras faz com que muitos moradores abandonem a zona rural e se instalem nas áreas urbanas. Essa transição faz com que cada vez mais a vegetação antes presente nas cidades, seja substituída ou alterada pelo desenvolvimento urbano. Apesar do desenvolvimento ser necessário, a perda da vegetação no cotidiano da população influencia diretamente na diminuição da qualidade de vida devido a diminuição do conforto, da estética ou da saúde, transformando as áreas urbanas em locais cada vez mais insalubres.

Neste sentido, a vegetação urbana, quando corretamente implantada, desempenha um conjunto importante de funções responsáveis pela melhoria da qualidade do ambiente, podendo minimizar o impacto ambiental causado pelos efeitos antrópicos da expansão das cidades (PIRES et al., 2010).

Porém, em muitas cidades brasileiras, a arborização viária foi estabelecida sem planejamento criterioso, onde, de maneira geral, apresentam indivíduos arbóreos de vegetação remanescente que sobreviveram ao desenvolvimento urbano, indivíduos de espécies inadequadas ao meio urbano ou indivíduos plantados de maneira incorreta.

Coleto, Müller e Wolski (2008) afirmam que para promover o desenvolvimento urbano também é indispensável planejar a arborização, com conhecimento prévio da situação existente, por meio de um inventário quali-quantitativo e do conhecimento das espécies que posteriormente poderão ser utilizadas.

Muito do conhecimento sobre a vegetação urbana no Brasil, é advindo dos inventários realizados geralmente por amostragens, onde a vegetação é identificada e quantificada, além de serem avaliadas quanto à condição estrutural e fitossanitária e as práticas de manejo (ANDRADE, 2002; BENATTI et. al, 2012; PARRY et al., 2012; SCHALLENBERGER; MACHADO, 2013; ALECAR et al., 2014)

Nesse contexto, os objetivos desta pesquisa foram realizar um inventário do patrimônio florístico do município de Itanhaém - SP e caracterizar a composição dos indivíduos arbóreos, arbustivos e palmeiras em termos quali-quantitativos.

\section{MATERIAL E MÉTODOS}

\section{Descrição da área de estudo}

O presente estudo foi desenvolvido no município de Itanhaém, litoral sul do Estado de São Paulo (Figura 1), sob as coordenadas 2411"1' Sul e 4647"22' Oeste, tendo como limite 
os municípios de São Vicente, Mongaguá, Pedro de Toledo, Peruíbe Juquitiba e São Paulo (IPT, 2012).

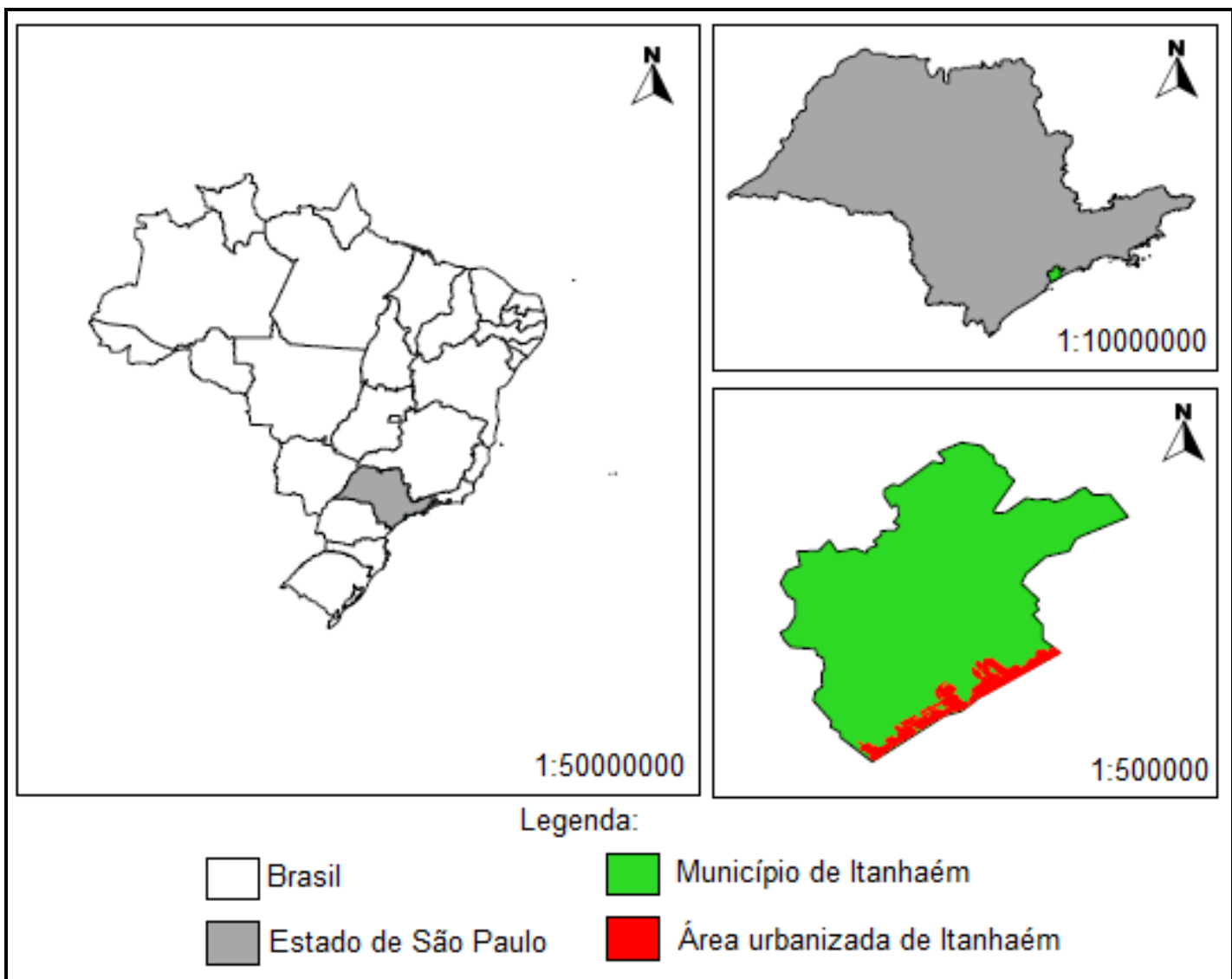

Figura 1. Localização do município de Itanhaém

Figure 1. Itanhaém city location

O clima predominante no município é do tipo Af, segundo a classificação de Köppen, caracterizado como clima tropical sem estação seca, com precipitação média mensal maior que $60 \mathrm{~mm}$ e temperatura média do mês mais frio igual a 18ㄷ (ALVAREZ et al., 2014). O município apresenta uma área territorial de 601,67 km² e uma área urbanizada de 40,8 km², com mais de 94.000 habitantes, sendo que mais de $99 \%$ da população está localizada na área urbana, a qual está dividida em 15 grandes regiões (Figura 2) que abrangem um total de 45 bairros (IBGE, 2014). 


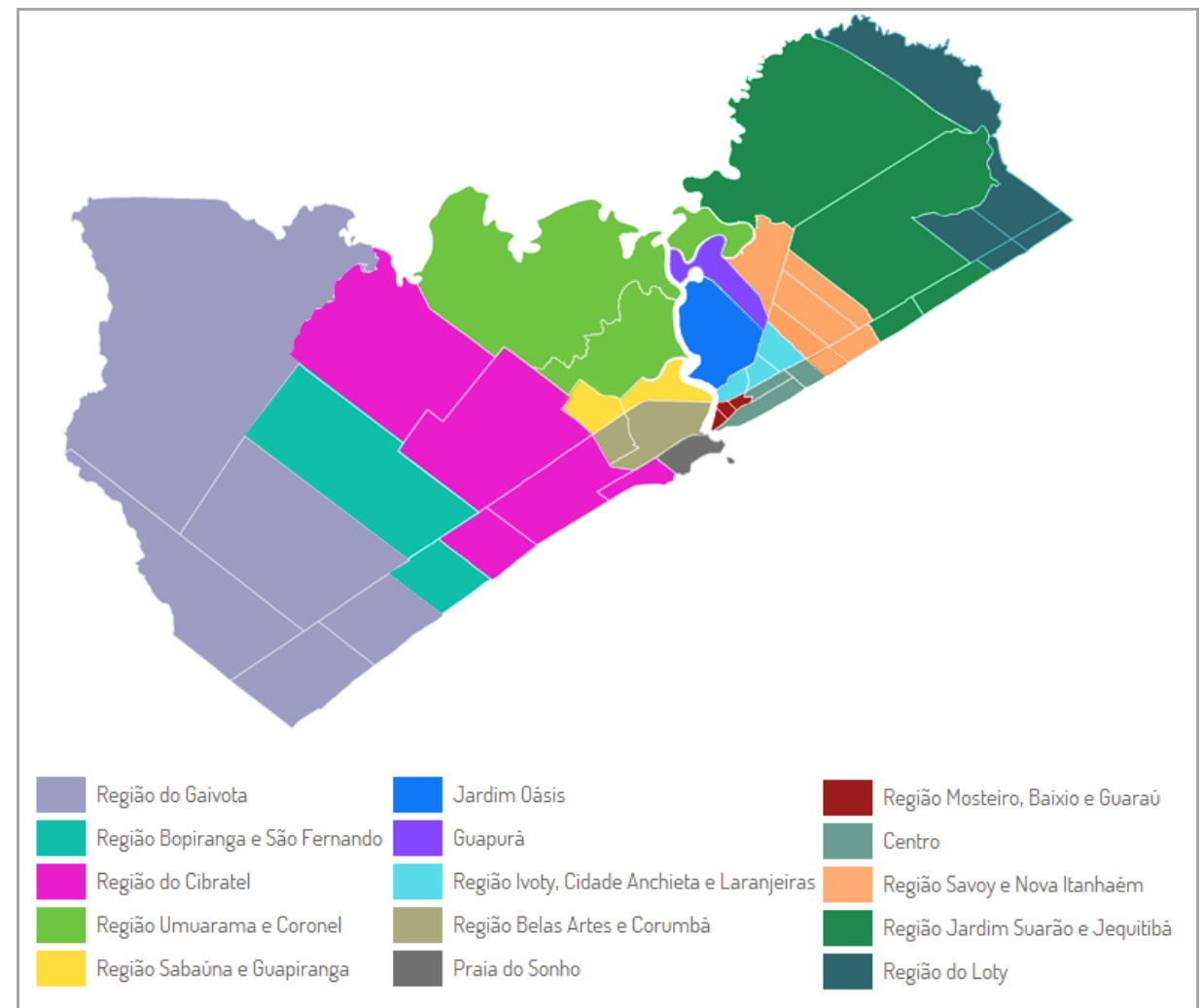

Fonte: Prefeitura Municipal de Itanhaém (2014)

Figura 2. Divisões e subdivisões das regionais do município de Itanhaém - SP

Figure 2. Divisions and subdivisions of the municipality regionals of Itanhaém - SP

\section{Coleta e análise dos dados}

O inventário quantitativo para cadastro florístico foi realizado por censo de todas as vias urbanizadas. Para isto, todo o município foi percorrido de carro, contabilizando as vias urbanizadas que, posteriormente, foram identificadas no software Google Earth® para mensuração da quilometragem de vias urbanizadas de cada regional.

Posteriormente, realizou-se a contagem dos indivíduos arbóreos, arbustivo e palmeiras de cada regional e a enumeração dos dados no software Excel® para determinação do número de árvores em cada regional e no total da cidade.

Pelo inventário quantitativo foram obtidas as seguintes informações: total de árvores, quilometragem de vias urbanizadas, número total de parcelas, número de árvores por quilômetro, número de habitantes, número de árvores/habitante, para cada regional e para o total da área urbanizada da cidade.

Para o inventário qualitativo utilizou-se o processo de amostragem estratificada, sendo utilizada a divisão administrativa do município em 15 regionais para a definição dos estratos. A forma da unidade amostral foi linear, variando seu comprimento entre 70-120 metros de acordo com a característica de cada regional, de modo que o comprimento caracterizasse a melhor 
distribuição de parcelas. As parcelas potenciais de cada regional foram numeradas e sorteadas aleatoriamente.

Para o cálculo da variância e do número de unidades amostrais necessárias utilizou-se o número de árvores por quilômetro de calçada que, de acordo com Milano (1984), é a variável que permite uma melhor homogeneização da variância.

As avaliações iniciavam-se pela amostragem piloto, onde eram sorteadas aproximadamente $10 \%$ das unidades amostrais " $n$ " de cada regional para cálculo de suficiência amostral e adicionadas novas unidades amostrais, até que o " $n$ " calculado se equivalesse ao "n" amostrado.

A intensidade amostral foi calculada com o nível de confiança de $95 \%$ e um erro de $15 \%$, a partir da variância amostral. As variáveis analisadas no inventário qualitativo foram adotadas tomando-se por base a metodologia proposta por Milano (1984) e adaptada por Bobrowski (2011). Para isto foram avaliados os seguintes itens: espécie, localização, altura da árvore $(\mathrm{H})$, circunferência à altura do peito (CAP), raios de copa (d - direita, e - esquerda, $r$ rua, c - calçada), condição do sistema radicular, condição estrutural e fitossanitária das árvores, necessidade de manejo e observações de caráter complementar (Figura 3).

\begin{tabular}{|c|c|c|c|c|c|c|c|c|c|c|c|c|c|c|c|c|c|}
\hline \multicolumn{18}{|c|}{ Inventário da Arborização viáia de Itanhaém - SP } \\
\hline \multicolumn{8}{|l|}{ Regional: } & \multicolumn{10}{|c|}{ Rua: } \\
\hline \multicolumn{8}{|l|}{ Lado: } & \multicolumn{10}{|c|}{ Número da Parcela: } \\
\hline \multirow[b]{2}{*}{ Espécie } & \multicolumn{4}{|c|}{ Posiçao na calçada } & \multirow[b]{2}{*}{$\mathrm{H}$} & \multirow[b]{2}{*}{$\mathrm{HF}$} & \multirow[b]{2}{*}{ CAP } & \multicolumn{4}{|c|}{ Copa } & \multirow[b]{2}{*}{ Hbif } & \multirow[b]{2}{*}{ ALC } & \multirow[b]{2}{*}{$\mathrm{R}$} & \multirow[b]{2}{*}{$\mathrm{C}$} & \multirow[b]{2}{*}{ NT } & \multirow[b]{2}{*}{ * } \\
\hline & Guia & Casa & Fiação & D.A. & & & & d & $\mathrm{e}$ & $\mathrm{r}$ & c & & & & & & \\
\hline & & & & & & & & & & & & & & & & & \\
\hline & & & & & & & & & & & & & & & & & \\
\hline & & & & & & & & & & & & & & & & & \\
\hline & & & & & & & & & & & & & & & & & \\
\hline & & & & & & & & & & & & & & & & & \\
\hline
\end{tabular}

Figura 3. Planilha de campo utilizada para o inventário qualitativo da arborização viária de Itanhaém - SP Figure 3. Field sheet used for the qualitative inventory of street afforestation of Itanhaem - SP

Para o parâmetro espécie, os indivíduos arbóreos foram identificados em campo a nível de gênero e família, sendo também avaliada a diversidade da arborização por meio do índice de diversidade de Shannon-Wiener.

\section{RESULTADOS E DISCUSSÃO}

\section{Análise quantitativa}

Em um total de 421,56 km de vias urbanizadas foram encontradas 18.128 árvores em vias urbanas no município (Tabela 1). 
Tabela 1. Dados quantitativos das regionais do município de Itanhaém

Table 1. Quantitative data of regionals of Itanhaém - SP

\begin{tabular}{lrrrrrr}
\hline Região & VU(km) & Narv & Narv/km & N.Hab. & Arv./hab. & Hab/VU \\
\hline Loty & 27,5 & 2473 & 89,9 & 4072 & 0,61 & 148,1 \\
Bopiranga & 28,39 & 1963 & 69,1 & 2651 & 0,74 & 93,4 \\
Umuarama & 6,48 & 443 & 68,4 & 6673 & 0,07 & 1029,8 \\
Suarão & 34,75 & 2018 & 58,1 & 5035 & 0,4 & 144,9 \\
Cibratel & 77,64 & 3914 & 50,4 & 4557 & 0,86 & 58,7 \\
Gaivota & 49,43 & 2366 & 47,9 & 5186 & 0,46 & 104,9 \\
Centro & 23,24 & 1011 & 43,5 & 3404 & 0,3 & 146,5 \\
Belas Artes e Corumbá & 32 & 912 & 28,5 & 9617 & 0,09 & 300,5 \\
Praia Dos Sonhos & 11,89 & 303 & 25,5 & 1383 & 0,22 & 116,3 \\
Oasis & 6,6 & 165 & 25 & 6704 & 0,02 & 1015,8 \\
Ivoty & 20,22 & 456 & 22,6 & 6630 & 0,07 & 327,9 \\
Savoy e Nova Itanhaém & 81,71 & 1777 & 21,7 & 16241 & 0,11 & 198,8 \\
Mosteiro & 5,79 & 90 & 15,5 & 1067 & 0,08 & 184,3 \\
Guapiranga e Sabaúna & 15,92 & 237 & 14,9 & 7481 & 0,03 & 469,9 \\
\hline Total & $\mathbf{4 2 1 , 5 6}$ & $\mathbf{1 8 1 2 8}$ & $\mathbf{4 3}$ & $\mathbf{8 0 7 0 1}$ & $\mathbf{0 , 2 2}$ & $\mathbf{1 9 1 , 4}$ \\
\hline Nota: VU (vias urbanizadas em quilômetros), Narv (número de árvores), QP (quantidade de parcelas \\
potenciais), Narv/km (número de árvores por quilômetro), N.Hab (número de habitantes em área urbanizada), \\
Arv/Hab (número de árvores por habitante em área urbanizada) e Hab/VU (número de habitantes por \\
quilômetro de vias urbanizadas)
\end{tabular}

Observou-se que em média foram encontradas 43 árvores por quilômetro, o que significa apenas 4,3 árvores por quadra, se considerada a quadra padrão de 100 metros de comprimento. O número de árvores por quilômetro encontrado no município de Itanhaém é inferior ao encontrado em Estância de Águas de São Pedro, onde foi encontrado 130 árvores por quilômetro (BORTOLETO et al., 2007), porém é superior ao número encontrado em Campos do Jordão, com 17,22 árvores por quilômetro (ANDRADE, 2002).

A regional com maior número de árvores foi Loty, com 89,9 árvores/km, enquanto a regional Guapiranga e Sabaúna apresentaram o menor número, 14,9 árvores $/ \mathrm{km}$. Este contraste pode ser explicado pelas características das regionais. A regional Loty está localizada no lado da praia, com urbanização mais antiga e poucas áreas verdes em sua extensão, mas com destaque para a arborização como principal fonte de vegetação dentre as vias. Já a regional Guapiranga, está localizada no lado do morro e apresenta uma urbanização recente, onde a maioria das casas apresenta área verde em seu próprio terreno, com jardins e quintais com vegetação preservada.

O Cibratel, apesar de ser a quinta regional em número de árvores por quilômetros $(50,4)$, foi a regional que apresentou maior número de árvores por habitante $(0,86)$. Tal dado pode ser justificado por esta ser a regional com menor número de habitantes por vias urbanizadas $(58,7)$ o que indica que a grande extensão da regional não é correspondente ao número de habitantes, pois esta é uma regional com grande número de casas de temporada ou de veraneio. 


\section{Suficiência amostral}

Para a realização do inventário qualitativo foram utilizadas as 15 regionais como estratos, sendo realizado o cálculo de números de parcelas a serem amostradas em cada regional (Tabela 2). Os dados apresentados demonstram que houve atendimento à suficiência amostral e que a amostragem realizada representa significativamente a arborização da cidade.

Tabela 2. Dados de suficiência amostral das regionais/estratos

Table 2. Sample sufficiency analysis of regional / stratum

\begin{tabular}{lrrrr}
\hline Regional & QPP & Ncalc & Na & Na(\%) \\
\hline Belas Artes & 199 & 19,001 & 23 & 11,6 \\
Cibratel & 243 & 15,91 & 16 & 6,6 \\
Centro & 157 & 13,879 & 15 & 9,6 \\
Loty & 179 & 13,218 & 13 & 7,3 \\
Ivoty & 127 & 13,128 & 15 & 11,8 \\
Praia dos Sonhos & 74 & 10,799 & 13 & 17,6 \\
Bopiranga & 105 & 10,098 & 10 & 9,5 \\
Guapiranga e Sabaúna & 105 & 10,098 & 10 & 9,5 \\
Mosteiro & 36 & 9,31 & 9 & 25,0 \\
Savoy & 290 & 8,561 & 11 & 3,8 \\
Suarão & 256 & 8,009 & 20 & 7,8 \\
Gaivota & 282 & 7,853 & 11 & 3,9 \\
Bopiranga & 155 & 5,683 & 8 & 5,2 \\
Umuarama & 40 & 4,404 & 6 & 15,0 \\
\hline Nota: QPP (quantidade de parcelas potenciais); Ncalc (número de parcelas \\
calculado); NA (número de parcelas amostradas); NA\% (número de \\
parcelas amostradas em porcentagem).
\end{tabular}

A regional Savoy foi a que apresentou maior número de parcelas potenciais (290), porém foi a que apresentou a menor porcentagem de parcelas amostradas (3,8\%). Já a regional Mosteiro, foi a que apresentou o menor número de parcelas potenciais (36), e a maior porcentagem de parcelas amostradas (25\%). Os valores apresentados indicam uma homogeneidade quanto ao número de árvores por parcelas na regional Savoy e uma heterogeneidade na regional Mosteiro, já que quanto maior a variância maior a porcentagem de parcelas a ser avaliada.

\section{Florística e proporção de táxons}

No município, foram avaliados de maneira qualitativa: 1265 indivíduos arbóreos em calçadas, classificados quanto à sua forma de vida (arbustos, árvores, palmeiras, cicas ou trepadeiras), identificados quanto à sua espécie e família botânica, sendo estes distribuídos em 109 espécies, 82 gêneros e 41 famílias botânicas (Tabela 3). 
Tabela 3. Distribuição quantitativa de cada espécie encontrada na arborização viária de Itanhaém (SP), segundo o nome comum, nome cientifico, família, origem $(\mathrm{O})$, frequência absoluta ( $F A$ ), frequência relativa (FR) e forma de vida (FV)

Table 3. Quantitative distribution of each species found in street afforestation of Itanhaém (SP), according to the common name, scientific name, family, origin $(\mathrm{O})$, absolute frequency (FA), relative frequency $(\mathrm{FR})$ and way of life $(\mathrm{FV})$

\begin{tabular}{|c|c|c|c|c|c|c|}
\hline Nome popular & Nome científico & Família & 0 & FA & FR & FV \\
\hline Chapéu-de-sol & Terminalia catappa L. & Combretaceae & A & 221 & 17,47 & Arv \\
\hline Ficus & Ficus benjamina L. & Moraceae & A & 212 & 16,76 & Arv \\
\hline Areca & Dypsis lutescens & Arecaceae & A & 142 & 11,23 & $\mathrm{Pal}$ \\
\hline Coqueiro & Cocos nucifera L. & Arecaceae & A & 92 & 7,27 & Pal \\
\hline Coqueiro-anão & Cocos nucifera L. (Nana) & Arecaceae & A & 35 & 2,77 & Pal \\
\hline Espirradeira & Nerium oleander $\mathrm{L}$. & Apocynaceae & A & 27 & 2,13 & Arb \\
\hline Flamboyant & Delonix regia (Bojer ex Hook.) Raf. & Fabaceae & A & 23 & 1,82 & Arv \\
\hline Ipê-amarelo & Handroanthus chrysotrichus (Mart. ex DC.) Mattos & Bignoniaceae & C & 22 & 1,74 & Arv \\
\hline Cassia-fistula & Cassia fistula $\mathrm{L}$. & Fabaceae & A & 19 & 1,50 & Arv \\
\hline Ipê-de-Jardim & Tecoma stans (L.) Juss. Ex kunth & Bignoniaceae & A & 19 & 1,50 & Arv \\
\hline Aroeira-pimenteira & Schinus terebinthifolius Raddi & Anacardiaceae & B & 18 & 1,42 & Arv \\
\hline Jerivá & Syagrus romanzoffiana (Cham.) Glassman & Arecaceae & $\mathrm{C}$ & 18 & 1,42 & Pal \\
\hline Pitangueira & Eugenia uniflora $\mathrm{L}$. & Myrtaceae & C & 17 & 1,34 & Arv \\
\hline Goiabeira & Psidium guajava L. & Myrtaceae & C & 16 & 1,26 & Arv \\
\hline Extremosa & Lagerstroemia indica (L.) Pers. & Lythraceae & A & 15 & 1,19 & Arv \\
\hline Hibisco & Hibiscus rosa-sinensis L. & Malvaceae & A & 15 & 1,19 & Arb \\
\hline Ipê & Handroanthus sp. Mattos & Bignoniaceae & - & 15 & 1,19 & Arv \\
\hline Murta & Murraya paniculata L. Jack & Rutaceae & A & 15 & 1,19 & Arv \\
\hline Espatódea & Spathodea campanulata P. Beauv. & Bignoniaceae & A & 14 & 1,11 & Arv \\
\hline Amoreira & Morus nigra L. & Moraceae & A & 13 & 1,03 & Arv \\
\hline Cica & Cycas revoluta Thunb. & Cicadaceae & A & 12 & 0,95 & $\mathrm{Cic}$ \\
\hline Oiti & Licania tomentosa (Benth.) Fritsch & Chrysobalanaceae & $\mathrm{C}$ & 12 & 0,95 & Arv \\
\hline Schefflera & Schefflera arborícola (Hayata) Merr. & Araliaceae & A & 12 & 0,95 & Arv \\
\hline Palm-fenix & Phoenix roebelinii O'Brien & Arecaceae & A & 11 & 0,87 & Pal \\
\hline Canudo-de-pito & Cassia bicapsularis L. & Fabaceae & C & 10 & 0,79 & Arv \\
\hline Palm-rabo-de-peixe & Caryota urens & Arecaceae & A & 10 & 0,79 & Pal \\
\hline Dracena & Dracena sp. & Asparagaceae & A & 9 & 0,71 & Arv \\
\hline Flamboyantzinho & Caesalpinia pulcherrima (L.) Sw. & Fabaceae & A & 9 & 0,71 & Arv \\
\hline Jaca & Artocarpus heterophyllus Lam. & Moraceae & A & 9 & 0,71 & Arv \\
\hline Abacateiro & Persea americana Mill. & Lauraceae & A & 7 & 0,55 & Arv \\
\hline Algodoeiro & Hibiscus pernambucensis Arruda & Malvaceae & B & 7 & 0,55 & Arv \\
\hline João-bolão & Syzygium cumini (L.) skeels & Myrtaceae & $A$ & 7 & 0,55 & Arv \\
\hline Bouganville & Bougainvillea spectabilis Willd. & Nyctaginaceae & B & 6 & 0,47 & Arb \\
\hline Chorão & Schinus molle L. & Anacardiaceae & B & 6 & 0,47 & Arv \\
\hline Cróton & Codiaeum variegatum (L.) Rumph. Ex A. Juss. & Euphorbiaceae & A & 6 & 0,47 & Arb \\
\hline Palheteira & Clitoria fairchildiana R.A.Howard & Fabaceae & B & 6 & 0,47 & Arv \\
\hline Cassia-grandis & Cassia grandis L. f. & Fabaceae & B & 5 & 0,40 & Arv \\
\hline Graviola & Annona muricata $\mathrm{L}$. & Moraceae & A & 5 & 0,40 & Arv \\
\hline Palm-real & Archontophoenix cunninghamii H. Wendl. \& Drude & Arecaceae & A & 5 & 0,40 & $\mathrm{Pal}$ \\
\hline Pata-de-vaca & Bauhinia forficata Link & Fabaceae & A & 5 & 0,40 & Arv \\
\hline
\end{tabular}




\begin{tabular}{|c|c|c|c|c|c|c|}
\hline Nome popular & Nome científico & Família & 0 & FA & FR & FV \\
\hline Abricó & Mimusops coriacea (A. DC.) Miq. & Sapotaceae & A & 4 & 0,32 & Arv \\
\hline Ameixinha-amarela & Eriobothrya japonica (Thunb.) Lindl. & Rosaceae & A & 4 & 0,32 & Arv \\
\hline Guanandi & Calophyllum brasiliense Cambess. & Clusiaceae & C & 4 & 0,32 & Arv \\
\hline Ipê-branco & Tabebuia roseoalba (Ridl.) Sandwith & Bignoniaceae & $\mathrm{B}$ & 4 & 0,32 & Arv \\
\hline Ipê-heptafila & Handroanthus heptaphyllus (Vell.) Mattos & Bignoniaceae & C & 4 & 0,32 & Arv \\
\hline Mangueira & Mangifera indica L. & Anacardiaceae & $A$ & 4 & 0,32 & Arv \\
\hline Morta & Não identificada & Não identificada & - & 4 & 0,32 & Arv \\
\hline Tipuana & Tipuana tipu (Benth.) Kuntz & Fabaceae & $A$ & 4 & 0,32 & Arv \\
\hline Acerola & Malpighia glabra L. & Malpighiaceae & $A$ & 3 & 0,24 & Arv \\
\hline Araçá & Psidium sp. L. & Myrtaceae & - & 3 & 0,24 & Arv \\
\hline Carambola & Averrhoa carambola L. & Oxalidaceae & A & 3 & 0,24 & Arv \\
\hline Dama-da-noite & Cestrum nocturnum $\mathrm{L}$. & Solanaceae & $A$ & 3 & 0,24 & Arb \\
\hline Eucalipto & Eucalyptus sp. & Myrtaceae & $A$ & 3 & 0,24 & Arv \\
\hline Fruta-do-conde & Annona squamosa L. & Annonaceae & $A$ & 3 & 0,24 & Arv \\
\hline Hibisco-vermelho & Malvaviscus arboreus Cav. & Malvaceae & $A$ & 3 & 0,24 & Arb \\
\hline Ipê-roxo-grande & Tabebuia impetiginosus (Mart. ex DC) Mattos & Bignoniaceae & $\mathrm{B}$ & 3 & 0,24 & Arv \\
\hline Laranjeira & Citrus sinensis & Rutaceae & A & 3 & 0,24 & Arv \\
\hline Pau-brasil & Caesalpinia echinata Lam. & Fabaceae & C & 3 & 0,24 & Arv \\
\hline Pingo-de-ouro & Duranta repens L. & Verbenaceae & $\mathrm{B}$ & 3 & 0,24 & Arb \\
\hline Pinhão-roxo & Jatropha gossypiifolia L. & Euphorbiaceae & A & 3 & 0,24 & Arb \\
\hline Quaresmeira & Tibouchina granulosa (Desr.) Cogn. & Melastomataceae & $\mathrm{C}$ & 3 & 0,24 & Arv \\
\hline Castanheira-da-praia & Pachira glabra Aubl. & Malvaceae & $\mathrm{B}$ & 2 & 0,16 & Arv \\
\hline Chapéu-de-napoleão & Thevetia peruviana (Pers.) K.Schum. & Apocynaceae & $A$ & 2 & 0,16 & Arb \\
\hline Coccoloba & Coccoloba uvifera L. & Polygonaceae & A & 2 & 0,16 & Arv \\
\hline Feijão-guandu & Cajanus cajan (L.) Huth & Fabaceae & $A$ & 2 & 0,16 & Arb \\
\hline Grumixama & Eugenia brasiliensis Lam. & Myrtaceae & $\mathrm{C}$ & 2 & 0,16 & Arv \\
\hline Ingá & Inga marginata Willd. & Fabaceae & $\mathrm{C}$ & 2 & 0,16 & Arv \\
\hline Ipê-amarelo-da-serra & Handroanthus albus (Cham.) Mattos & Bignoniaceae & C & 2 & 0,16 & Arv \\
\hline Jasmim-manga & Plumeria rubra L. & Apocynaceae & A & 2 & 0,16 & Arv \\
\hline Leucena & Leucaena leucocephala (Lam.) de Wit & Fabaceae & A & 2 & 0,16 & Arv \\
\hline Ligustrum & Ligustrum Iucidium W.T.Aiton & Oleaceae & A & 2 & 0,16 & Arv \\
\hline Limão & Citrus sp. & Rutaceae & $A$ & 2 & 0,16 & Arv \\
\hline Mil-cores & Breynia disticha J.R. Forst. \& G. Forst. & Phyllanthaceae & A & 2 & 0,16 & Arb \\
\hline Mulungu & Erythrina speciosa Andrews & Fabaceae & C & 2 & 0,16 & Arv \\
\hline Palm-leque & Washingtonia robusta $\mathrm{H}$. Wendl. & Arecaceae & A & 2 & 0,16 & Pal \\
\hline Podocarpus & Podocarpus sp. & Podocarpaceae & A & 2 & 0,16 & Arv \\
\hline Sibipiruna & $\begin{array}{l}\text { Caesalpinia pluviosa var. peltophoroides (Benth.) } \\
\text { G.P.Lewis }\end{array}$ & Fabaceae & $\mathrm{C}$ & 2 & 0,16 & Arv \\
\hline Não Identificada & Não identificada & Não identificada & - & 2 & 0,16 & Arv \\
\hline Alamanda & Allamanda cathartica L. & Apocynaceae & C & 1 & 0,08 & Arb \\
\hline Angico & Parapiptadenia rigida (Benth.) Brenan & Fabaceae & $\mathrm{C}$ & 1 & 0,08 & Arv \\
\hline Azaléia & Rhododendron simsii Planch. & Ericaceae & A & 1 & 0,08 & Arb \\
\hline Boldo & Plectranthus barbatus Andr. & Lamiaceae & $A$ & 1 & 0,08 & Arb \\
\hline Brasileirinho & Erythrina variegata L. & Fabaceae & $A$ & 1 & 0,08 & Arv \\
\hline Cactus & Nopalea cochenillifera (L.) Salm-Dyck & Cactaceae & $A$ & 1 & 0,08 & Arb \\
\hline
\end{tabular}




\begin{tabular}{|c|c|c|c|c|c|c|}
\hline Nome popular & Nome científico & Família & 0 & FA & FR & FV \\
\hline Castanheira & Bertholletia excelsa Bonpl. & Lecythidaceae & $\mathrm{B}$ & 1 & 0,08 & Arv \\
\hline Cipreste & Cupressus sempervirens L. & Cupressasseae & A & 1 & 0,08 & Arv \\
\hline Corticeira & Erytrina crista-galli L. & Fabaceae & $\mathrm{B}$ & 1 & 0,08 & Arv \\
\hline Embiratanha & Pseudobombax marginatum (A.St.-Hil.) A. Robyns & Malvaceae & $\mathrm{B}$ & 1 & 0,08 & Arv \\
\hline Fruta-pão & Artocarpus altilis (Parkinson) Fosberg & Moraceae & A & 1 & 0,08 & Arv \\
\hline Hibisco-crespo & Hibiscus schizopetalus (Dyer ex Mast.) Hook, f. & Malvaceae & A & 1 & 0,08 & Arb \\
\hline Ingá-bravo & $\begin{array}{l}\text { Lonchocarpus cultratus (Vell.) A.M.G.Azevedo \& } \\
\text { H.C.Lima }\end{array}$ & Fabaceae & $\mathrm{C}$ & 1 & 0,08 & Arv \\
\hline Ipomea & $\begin{array}{l}\text { Ipomea carnea subsp. Fistulosa (Mart. Ex Chisy) } \\
\text { D.F.Austin }\end{array}$ & Convolvulaceae & $\mathrm{B}$ & 1 & 0,08 & Arb \\
\hline Ixora-vermelha & Ixora chinensis Lam. & Rubiaceae & A & 1 & 0,08 & Arb \\
\hline Jambo-rosa & Syzygium samarangense (BI.) Merr. Et Perry & Myrtaceae & A & 1 & 0,08 & Arv \\
\hline Jatobá & Hymenaea courbaril L. var. courbaril & Fabaceae & C & 1 & 0,08 & Arv \\
\hline Jurubeba & Solanum paniculatum L. & Solanaceae & $B$ & 1 & 0,08 & Arv \\
\hline Limão-tahiti & Citrus $x$ limon & Rutaceae & $E$ & 1 & 0,08 & Arv \\
\hline Melastomataceae & Não identificada & Melastomataceae & - & 1 & 0,08 & Arv \\
\hline Mussaenda-rosa & Mussaenda X philippica & Rubiaceae & A & 1 & 0,08 & Arb \\
\hline Paineira & Chorisia speciosa A. St.-Hil. & Malvaceae & $\mathrm{B}$ & 1 & 0,08 & Arv \\
\hline Palm-triangular & Dypsis decaryi & Arecaceae & $A$ & 1 & 0,08 & Pal \\
\hline Pokam & Citrus reticulata Blanco & Rutaceae & A & 1 & 0,08 & Arv \\
\hline Roxinho & Euphorbia cotinifolia L. & Euphorbiaceae & $A$ & 1 & 0,08 & Arb \\
\hline Seriguela & Spondias purpurea L. & Anacardiaceae & $\mathrm{B}$ & 1 & 0,08 & Arv \\
\hline Tamareira & Phoenix dactylifera L. & Arecaceae & A & 1 & 0,08 & Pal \\
\hline Tangerina & Citrus reticulata & Rutaceae & A & 1 & 0,08 & Arv \\
\hline $\begin{array}{l}\text { Thunbergia-azul- } \\
\text { arbustiva }\end{array}$ & Thurbergia erecta (Benth.) T. Anderson & Acanthaceae & $A$ & 1 & 0,08 & Arb \\
\hline Vacum & $\begin{array}{l}\text { Allophylus edulis (A. St.-Hil., Cambess. \& A. Juss.) } \\
\text { Hieron. ex Niederl. }\end{array}$ & Sapindaceae & B & 1 & 0,08 & Arv \\
\hline Viuvinha & Petrea subserrata Cham. & Verbenaceae & $\mathrm{B}$ & 1 & 0,08 & Trp \\
\hline
\end{tabular}

Nota: A - exótica; B - exótica da Mata Atlântica e nativa do Brasil; C - nativa da Mata Atlântica; Arv - Árvore; Arb Arbussto; Pal - Palmeira; Cic - Cicadaceae; Trp - Trepadeira.

O número de espécies encontradas na arborização (109) foi superior ao encontrado em cidades como Goiandira (GO) com 105 espécies (PIRES et al., 2010); 60 espécies encontradas por Coleto, Müller e Wolski (2008) em Sete se Setembro (RS) e 113 em Maringá (SAMPAIO; DE ANGELIS, 2008).

Dentre as espécies encontradas na arborização viária, 60,5\% (66 espécies) são de origem exótica, 17,4\% (19 espécies) são de origem nativa do Brasil e 17,4\% (19 espécies) são originárias do Bioma Mata Atlântica, ou seja, nativas para a área de estudo. A presença de espécies exóticas é muito comum na arborização viária de muitas cidades. Para Paiva (2009), a predominância de espécies exóticas na composição da arborização viária é regra geral na maioria das cidades brasileiras. Em Cafeara - PR as espécies exóticas foram responsáveis por $68 \%$ da vegetação que compunha a arborização viária (LOCASTRO et al., 2014) e em Godoy Moreira - PR, 76\% das espécies são de origem exótica (MIRANDA et al., 2015). 
O índice de diversidade de Shannon encontrado para este inventário da cidade foi igual a 3,34, valor inferior ao encontrado por Bortoleto et al. (2007) em Estância Águas de São Pedro, onde o índice encontrado foi de 3,90, porém o índice acima de 3 é considerado um índice com uma boa diversidade de espécies.

A adequada diversidade de espécies pode ser confirmada pela frequência relativa das espécies, pois apesar da expressividade das espécies mais frequentes, Terminalia catappa, Ficus benajmina e Dypsis lutescenes, e Cocos nucifera, incluindo a sua variedade anã, representarem aproximadamente 50\% dos indivíduos, as demais espécies apresentaram certa equidade, com uma frequência que variou entre 0,8 e $2.1 \%$.

A proporção de táxons foi analisada para família, gênero e espécie, onde foram selecionados os 15 táxons de maior frequência (Tabela 4).

Tabela 4. Proporção dos 15 táxons de maior frequência

Table 4. Proportion of the 15 higher frequency taxons

\begin{tabular}{|c|c|c|c|c|c|c|c|c|}
\hline Família & $\mathbf{N}$ & $\begin{array}{l}\text { FR } \\
(\%)\end{array}$ & Gênero & $\mathbf{N}$ & $\begin{array}{l}\text { FR } \\
(\%)\end{array}$ & Espécie & $\mathbf{N}$ & $\begin{array}{l}\text { FR } \\
(\%)\end{array}$ \\
\hline Arecaceae & 317 & 25,06 & Terminalia & 221 & 17,47 & Terminalia catappa & 221 & 17,47 \\
\hline Moraceae & 240 & 18,97 & Ficus & 212 & 16,76 & Ficus benjamina & 212 & 16,76 \\
\hline Combretaceae & 221 & 17,47 & Dypsis & 143 & 11,30 & Dypsis lutescens & 142 & 11,23 \\
\hline Fabaceae & 98 & 7,75 & Cocos & 127 & 10,04 & Cocos nucifera & 92 & 7,27 \\
\hline Bignoniaceae & 83 & 6,56 & Handroanthus & 50 & 3,95 & Cocos nucifera (nana) & 35 & 2,77 \\
\hline Myrtaceae & 49 & 3,87 & Cassia & 34 & 2,69 & Nerium oleander & 27 & 2,13 \\
\hline Apocynaceae & 32 & 2,53 & Nerium & 27 & 2,13 & Delonix regia & 23 & 1,82 \\
\hline Malvaceae & 30 & 2,37 & Schinus & 24 & 1,90 & Handroanthus chrysotrichus & 22 & 1,74 \\
\hline Anacardiaceae & 29 & 2,29 & Delonix & 23 & 1,82 & Cassia fistula & 19 & 1,50 \\
\hline Rutaceae & 23 & 1,82 & Hibiscus & 23 & 1,82 & Tecoma stans & 19 & 1,50 \\
\hline Lythraceae & 15 & 1,19 & Eugenia & 19 & 1,50 & Schinus terebinthifolia & 18 & 1,42 \\
\hline Araliaceae & 12 & 0,95 & Psidium & 19 & 1,50 & Syagrus romanzoffiana & 18 & 1,42 \\
\hline Chrysobalanaceae & 12 & 0,95 & Tecoma & 19 & 1,50 & Eugenia uniflora & 17 & 1,34 \\
\hline Cicadaceae & 12 & 0,95 & Syagrus & 18 & 1,42 & Psidium guajava & 16 & 1,26 \\
\hline Euphorbiaceae & 10 & 0,79 & Lagerstroemia & 15 & 1,19 & Handroanthus sp. & 15 & 1,19 \\
\hline
\end{tabular}

As quinze famílias mais frequentes somaram $37,5 \%$ do total de famílias e representam 93\% dos indivíduos amostrados, e nenhuma superou o limite recomendado por Santamour Júnior (1990), que recomenda que a frequência dos táxons de espécie, gênero e família não devem ultrapassar 10, 20 e $30 \%$ do número de indivíduos da paisagem urbana, respectivamente, pois assim assegura-se o máximo de proteção contra o ataque de pragas e doenças. Entretanto, a adaptabilidade das espécies e a longevidade potencial são fatores tão ou mais importantes que a diversidade, pois algumas espécies, mesmo em pequena frequência, podem apresentar problemas fitossanitários que comprometem sua manutenção no meio urbano (RAUPP; CUMMING; RAUPP, 2006).

A família que apresentou maior frequência foi Arecaceae com 317 indivíduos e 25\% do total de indivíduos amostrados, representando 10 espécies classificadas como Palms. No 
viveiro da prefeitura, não são disponibilizados indivíduos desta família, portanto, todos os indivíduos localizados na malha viária foram plantados pelos próprios moradores, que provavelmente ligam erroneamente a região litorânea exclusivamente a este tipo de vegetação.

Em Altamira (PA), a família Arecaceae foi a segunda família de maior ocorrência na arborização urbana, sendo representada também por 10 espécies (PARRY et al., 2012); em Petrolina (PE) a família Arecaceae também foi a segunda de maior frequência e representou $14 \%$ dos indivíduos amostrados do centro da cidade (ALVAREZ et al., 2009). A segunda família mais frequente foi a Moraceae, com duas espécies representando 18,97\% dos indivíduos. Em Sorocaba - SP, em inventário da arborização viária, a família Moraceae apresentou 6 indivíduos, e apresentou um total de 10,6\% dos indivíduos arbóreos. (CARDOSO-LEITE et al., 2014).

As quinze espécies mais frequentes representaram 14\% do total de espécies e $70,8 \%$ dos indivíduos amostrados. As espécies Terminalia catappa (chapéu-de-sol) e Ficus benjamina (ficus) apresentaram frequência de 17,47\% e 16,76\% respectivamente. As frequências de ambas as espécies são superiores à recomendada por Grey e Deneke (1986), onde afirmaram que a frequência por espécie não pode ultrapassar 15\%. Já para classificação de Santamour Júnior (1990), a lista de espécies que superam a frequência desejada é maior, já que para esse autor a frequência de cada espécie não deve exceder a 10\%, nesse caso inclui-se também a espécie Dypsis lutescens (areca), já que a mesma apresenta $11,3 \%$ dos indivíduos.

A espécie de maior frequência foi Terminalia catappa, representada por 221 indivíduos. A espécie é uma das poucas do município que atinge grande porte, e por isso se tornam muito atraentes para a população, devido à sombra que pode proporcionar. A espécie também foi a de maior ocorrência em uma avaliação da arborização de ruas e praças de Salvador - BA, sendo representada por 10,8\% dos indivíduos (GÓES; OLIVEIRA, 2011); a segunda de maior ocorrência na arborização urbana do bairro Santa Quitéria, em Timon - MA (MORAES; MACHADO, 2014); e foi a quarta espécie de maior ocorrência no município de Pirapora - SP (BENATTI et al., 2012).

A segunda espécie mais frequente foi Ficus benjamina, representada por 212 indivíduos. Esta espécie vem sendo plantada pelos moradores, por ser a espécie comercial de mais fácil acesso no município, sendo geralmente aplicada, sem instruções, a técnica de topiaria, causando sérios danos aos exemplares desta espécie. A mesma espécie foi citada por Benatti et al. (2012), como a segunda espécie de maior ocorrência, representando 10,4\% dos indivíduos encontrados na arborização urbana de Salto do Pirapora -SP. Na cidade de São João do Rio do Peixe - PB a mesma espécie foi indicada como segunda de maior ocorrência com 19,87\% dos indivíduos amostrados (ALENCAR et al., 2014). 


\section{Análise qualitativa}

Dos 1265 indivíduos avaliados qualitativamente, 678 (54\%) encontravam-se em boas condições estruturais e fitossanitárias, 327 (26\%) apresentavam condições satisfatórias, 240 (19\%) apresentavam-se em condições ruins e 20 indivíduos (1\%) estavam mortos (Figura 4).
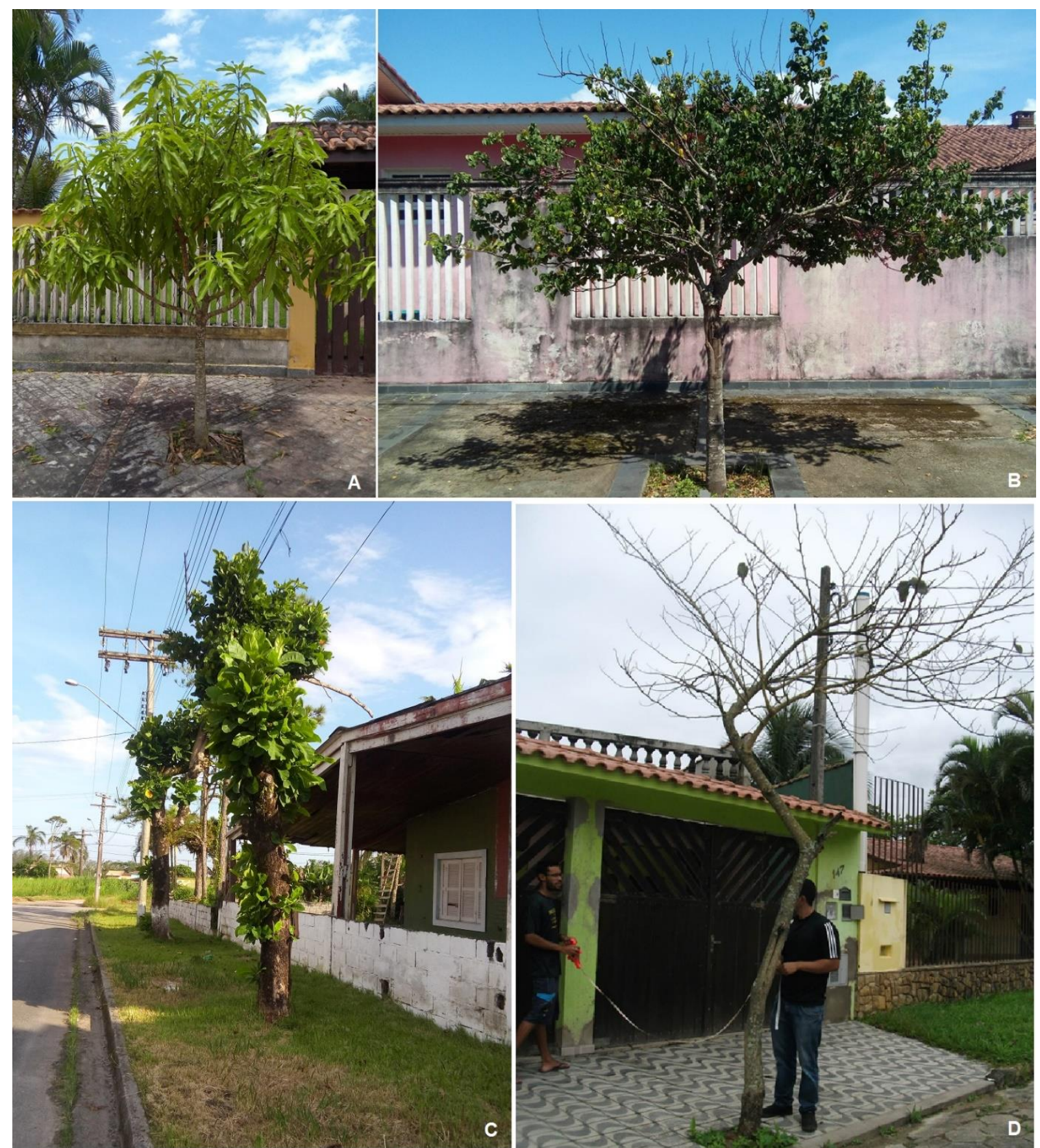

Figura 4. Classificação dos indivíduos arbóreos quanto às condições estruturais e fitossanitárias: (A) indivíduo em boas condições; (B) em condições satisfatórias; (C) em condições ruins; (D) morta

Figure 4. Classification of individual trees as structural and phytosanitary conditions. (A) individual in good condition; (B) in satisfactory condition; (C) in bad conditions; (D) dead 
A porcentagem de indivíduos em boas condições foi superior à encontrada em Aracaju-SE, onde apenas 38\% dos indivíduos apresentavam esta classificação (SANTOS et al., 2015); e também superior ao encontrado em Maringá, onde apenas 18,2\% dos indivíduos foram classificados em boas condições estruturais e fitossanitárias (SAMPAIO; DE ANGELIS, 2008). Isto pode ser um indicativo da moderada adaptabilidade das espécies às condições da arborização de vias públicas da cidade de Itanhaém.

Quanto à condição das raízes, 811 indivíduos (64\%) apresentaram raiz profunda, 202 (16\%) apresentaram raiz pouco superficial e 252 (20\%) apresentaram raiz superficial (Figura 5). A avaliação das raízes nas calçadas de Pirapora-SP também demonstrou que aproximadamente $70 \%$ das Arvs não interferiam no passeio, garantindo que a arborização pode se adequar ao meio urbano mediante um planejamento (BENATTI et al., 2012). Estes dados indicam uma maior facilidade das raízes em penetrar no solo local, desde que disponibilizado o espaço adequado para o desenvolvimento das mesmas.

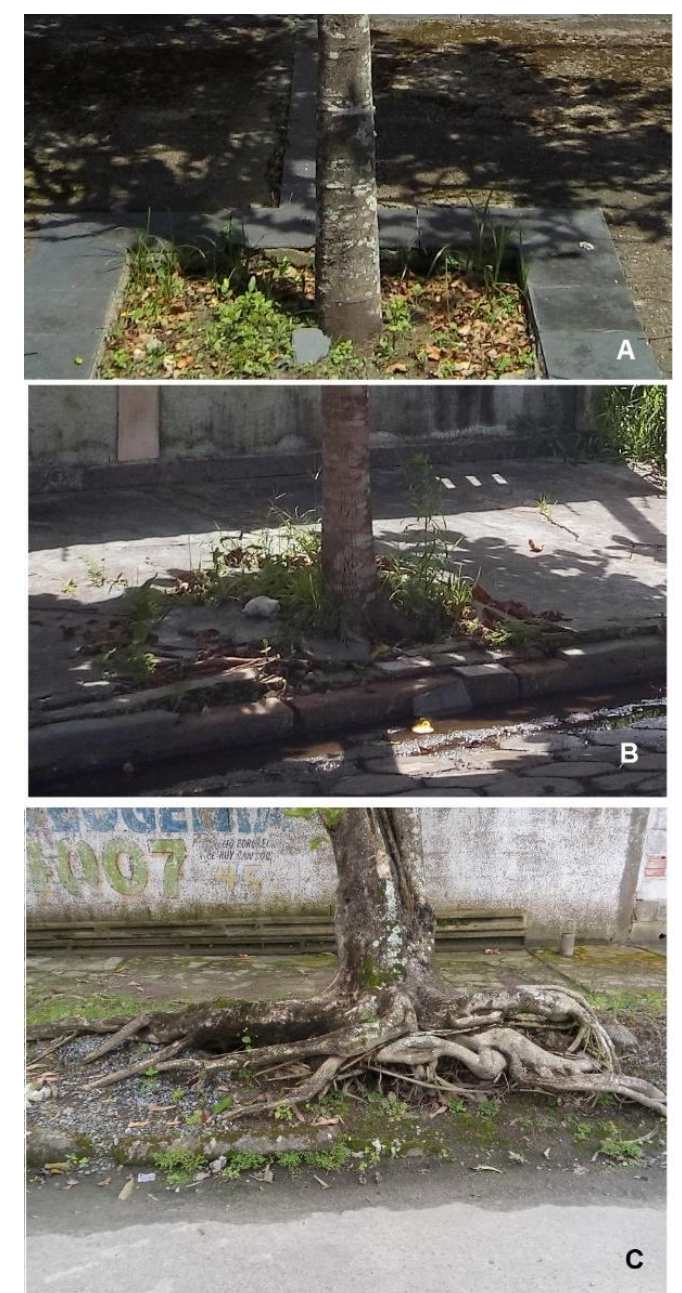

Figura 5. Classificação dos indivíduos arbóreos quanto à condição de raiz: (A) indivíduo com raiz profunda; (B) com raiz pouco superfial; (C) com raiz superficial

Figure 5. Classification of individual trees as the root condition. (A) individual with deep roots; (B) with little superfial root; (C) with shallow root 
Quanto à distribuição dendrométrica dos indivíduos avaliados, a maioria apresentou DAP entre 0 e $10 \mathrm{~cm}$, com média de 16,8 cm de DAP, e altura média de 4,6 m (Figura 6).

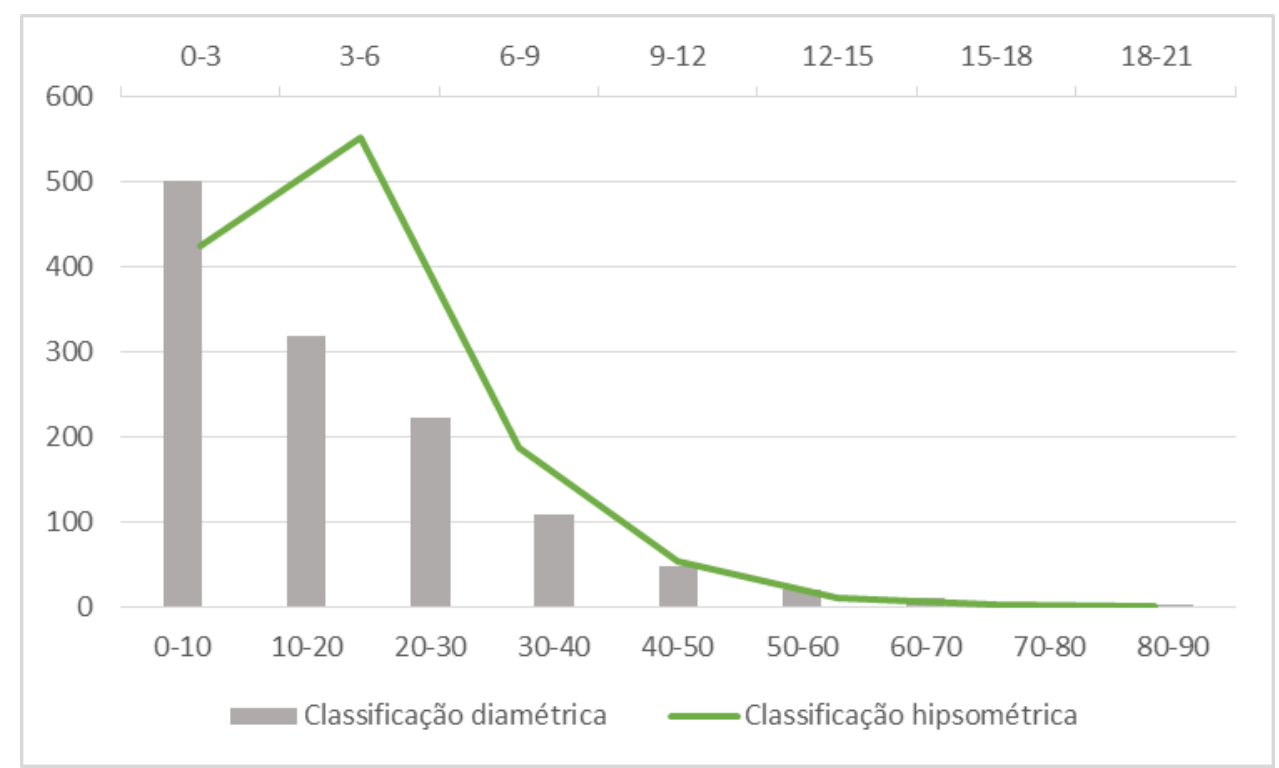

Figura 6. Relação dendrométrica da arborização viária do município de Itanhaém-SP quanto a classificação diamétrica e hipsométrica

Figure 6. Dendrometric relation graph of street tree in the city of Itanhaém-SP about the diametric and hypsometric classifications

Os resultados da classificação dendrométrica demonstram que a vegetação é composta por indivíduos jovens e/ou de pequeno a médio porte, com a maioria dos indivíduos entre as classes de 0-20 cm de DAP (64,7\%), e entre 0 e 6 metros de altura $(76,4 \%)$. Resultados similares foram encontrados na arborização viária de Araçoiaba da Serra (SP), onde aproximadamente $60 \%$ dos indivíduos apresentavam menos de 5 metros de altura e aproximadamente $80 \%$ apresentava DAP entre 5 e $35 \mathrm{~cm}$ (GRACIANO-SILVA; CARDOSOLEITE; TONELLO, 2014). Estes dados demonstram que a arborização da cidade se apresenta jovem, com pequenas dimensões, o que pode indicar que a oferta de benefícios ambientais ainda não atingiu seu limite máximo, podendo ser potencializado com ações colaborativas, como plantios participativos que estreitem a relação entre moradores e a prefeitura, para que haja um desenvolvimento de sucesso da vegetação.

Quanto à necessidade de manejo (Figura 7), a maior parte dos indivíduos não mostrou necessidade de intervenção (49,8\%), sendo a poda leve o tratamento indicado para 352 indivíduos (27\%), controle de doenças para 146 (11,5\%), a remoção foi indicada para 80 indivíduos $(4,3 \%)$, poda pesada para $43(3,4 \%)$, reparos de danos físicos e controle de pragas somaram $2 \%$. 


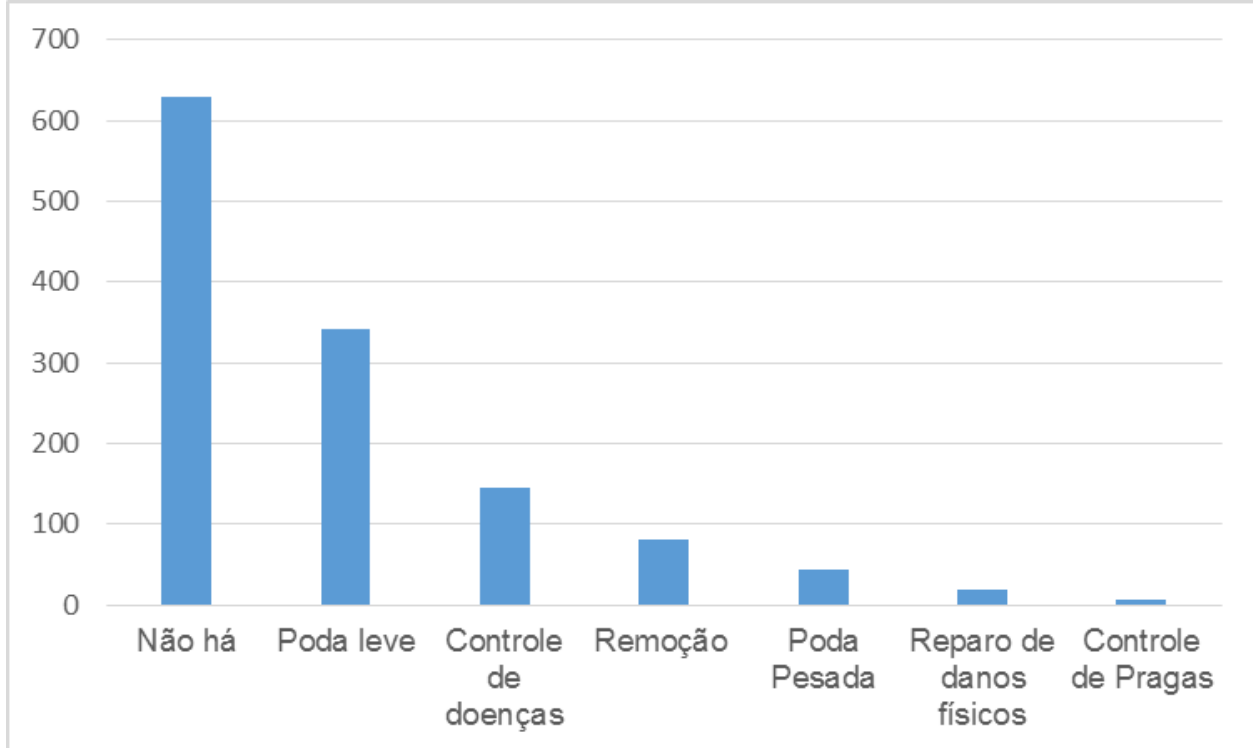

Figura 7. Principais necessidades de manejo da arborização viária de Itanhaém - SP Figure 7. Top management needs of street afforestation in Itanhaém - SP

A pouca necessidade de manejo pode ser consequência do número de indivíduos jovens ou de pequeno e médio porte, diminuindo a necessidade de intervenção quanto aos conflitos físicos com o meio urbano e a grande diversidade de espécies, que provavelmente fez diminuir as necessidades de intervenção quanto a pragas ou doenças em grande escala.

A infestação por cupim, comprometendo a estrutura do indivíduo arbóreo, foi responsável por aproximadamente $57 \%$ dos indivíduos com necessidade de remoção. $\mathrm{O}$ número é inferior aos dados de Maringá onde $13 \%$ da população amostrada, apresentou presença de cupins (SAMPAIO; DE ANGELIS, 2008).

Apesar da infestação por cupim ter baixa frequência, ocorrendo em apenas 3,6\% da população amostrada, ela é a maior causa de supressão, necessitando maior cuidado com seu controle.

\section{CONCLUSÃO}

Foram encontrados 18.128 indivíduos vegetais implantados em calçadas na área urbanizada de Itanhaém-SP. O bairro com maior número de Arvs por quilometro foi 0 Bopiranga com 89,9 Arvs e aquele com maior número de Arvs por habitantes foi o bairro Cibratel, com 0,86 Arvs.

As espécies mais frequentes foram Terminalia catappa (chapéu-de-sol), Ficus benjamina (ficus) e Dypsis lutescens (areca), e a família mais frequente foi Arecacea, com um índice de diversidade de Shannon de 3,34, garantindo uma boa diversidade de espécies da arborização viária. 
Os indivíduos encontrados no município são em sua maioria jovens e/ou de pequeno e médio porte, sendo que $76,4 \%$ dos indivíduos não ultrapassam 6 metros de altura, e $64,7 \%$ tem DAP inferior a $20 \mathrm{~cm}$.

A maioria dos indivíduos apresentou boas condições estruturais e fitossanitárias (54\%) e raízes profundas (64\%). Estas características contribuem para boa formação dos indivíduos nas ruas diminuindo as necessidades de manejo, já que 49,8\% dos indivíduos não apresentaram necessidade de manejo e a poda leve é necessária em $27 \%$ dos casos.

\section{REFERÊNCIAS}

ALENCAR, L.S.; SOUTO, P.C.; MOREIRA, F.T.A.; SOUTO, J.S.; BORGES, C.H.A. Inventário quali-quantitativo da arborização urbana em São João do Rio do Peixe - PB. Agropecuária Científica no Semi-árido, Campina Grande, PB, v. 10, n.2, p.117-124, abr-jun, 2014

ALVAREZ, C.A.; STAPE, J.L.; SENTELHAS, P.C.; GONÇALVES, J.L.M.; SPAROVEK, G. Köppen's climate classification map for Brazil. Meteorologische Zeitschrift, Stuttgart, v.22, n. 6, p.711-728, 2014.

ALVAREZ, I. A.; OLIVEIRA, U.R.; CARVALHO, J.C.L.; TAURA, T.A. Uso de geotecnologias para subsidiar planos de ação da arborização viária do centro de Petrolina-PE. In. CONGRESSO BRASILEIRO DE ARBORIZAÇÃO URBANA: DIVERSIDADE NA FLORESTA E NA CIDADE, 2009. Anais... Rio Branco: Sociedade Brasileira de Arborização Urbana. 2009.

ANDRADE, T.O. Inventário e análise da arborização viária da estância turística de Campos do Jordão, SP. Piracicaba - SP, 2002, 129 f. Dissertação (Mestrado em Agronomia) - Escola Superior de Agricultura Luiz de Queiroz da Universidade de São Paulo, Piracicaba, 2002.

BENATTI, D.P.; TONELLO, K.C.; ADRIANO JÚNIOR, F.C.; SILVA, J.M.S.; OLIVEIRA, I.R.; ROLIM, E.N.; FERRAZ, D.L. INVENTÁRIO ARBÓREO-URBANO DO MUNICÍPIO DE SALTO DE PIRAPORA, SP. Revista Arv, Viçosa, MG, v. 36, n. 5, p.887-894, 2012.

BORTOLETO, S.; SILVA FILHO, D.F.; SOUZA, V.C.; FERREIRA, M.A.P.; POLIZZEL, J.L.; RIBEIRO, R.C.S. COMPOSIÇÃO E DISTRIBUIÇÃO DA ARBORIZAÇÃO VIÁRIA DA ESTÂNCIA DE ÁGUAS DE SÃO PEDRO-SP. Revista da Sociedade Brasileira de Arborização Urbana, Piracicaba, SP, v.2, n.3, p. 32-46, 2007.

BOBROWSKI, R. ESTRUTURA E DINÂMICA DA ARBORIZAÇÃO DE RUAS DE CURITIBA, PARANÁ, NO PERÍODO 1984-2010. Curitiba - PR, 2011, 145 f. Dissertação (Mestrado em Engenharia Florestal) - Universidade Federal do Paraná, Curitiba, 2011.

CARDOSO-LEITE, E.; FARIA, L.C.; CAPELO, F.F.M.; TONELLO K.C.; CASTELLO, A.C.D. COMPOSIÇÃO FLORÍSTICA DA ARBORIZAÇÃO URBANA DE SOROCABA/SP, BRASIL. Revista da Sociedade Brasileira de Arborização urbana, Piracicaba, SP, v.9, n.1, p. 133150, 2014. 
COLETO, E.P.; MÜLLER, N.G.; WOLSKI, S.S. DIAGNÓSTICO DA ARBORIZAÇÃO DAS VIAS PÚBLICAS DO MUNICÍPIO DE SETE DE SETEMBRO - RS. Revista da Sociedade Brasileira de Arborização Urbana, Piracicaba, SP, v.3, n.2, p. 110-122, jun. 2008.

GÓES, G.S.; OLIVEIRA, M.Z.A. ARBORIZAÇÃO DE RUAS E PRAÇAS EM SALVADOR, BAHIA. Revista da Sociedade Brasileira de Arborização Urbana, Piracicaba, SP, v. 6, n.2, p.22-43, 2011.

GRACIANO-SILVA, T.; CARDOSO-LEITE, E.; TONELLO, K.C. INVENTÁRIO DA ARBORIZAÇÃO URBANA NO MUNICÍPIO DE ARAÇOIABA DA SERRA, SP. Revista da Sociedade Brasileira de Arborização Urbana, Piracicaba, SP, v.9, n.4, p.151-169, 2014.

GREY, G. W.; DENEKE, F. J. Urban Forestry. 2.ed. New York: J. Wiley, 1986. 199p.

INSTITUTO BRASILEIRO DE GEOGRAFIA E ESTATISTICA - IBGE. Censo. 2010, 1 p. Disponível em: <http://censo2010.ibge.gov.br/noticiascenso?view=noticia\&id=3\&idnoticia= 1766\&busca=1\&t=censo-2010-populacao-brasil-190- 732-694-pessoas $>$. Acesso em: 07 de jan. 2014.

INSTITUTO DE PESQUISAS TECNOLÓGICAS DO ESTADO DE SÃO PAULO - IPT. PREFEITURA MUNICIPAL DE ITANHAÉM - PMI. Atlas Ambiental do município de Itanhaém - 2012. São Paulo: Imprensa Oficial, 2012, 92p.

LOCASTRO, J.K.; RASBOLD, G.G.; PERREIRA, J.S.R.; SOARES, B.; CAXAMBU, M.G. CENSO DA ARBORIZAÇÃO URBANA DO MUNICÍPIO DE CAFEARA, PARANÁ. Revista da Sociedade Brasileira de Arborização Urbana, Piracicaba, SP, v.9, n.3, p. 122-140, 2014.

MILANO, M S. Avaliação e Análise da Arborização de Ruas de Curitiba-PR. Dissertação (Mestrado em Ciências Florestais), UFPR, Curitiba, Paraná, 1984. 130 p.

MIRANDA, Y.C.; MACHADO, M.S.; SILVA, L.S.; ESTEVAM, R.; MARTINS NETO, F.F.; CAXAMBU, M.G. ANÁLISE QUALI-QUANTITATIVA DA ARBORIZAÇÃO DE RUAS DO MUNICÍPIO DE GODOY MOREIRA - PR. Revista da Sociedade Brasileira de Arborização Urbana, Piracicaba, SP, v.10, n.1, p.71-81, 2015.

MORAES, L. A.; MACHADO, R. R. B. A arborização urbana do município de Timon/MA: Inventário, diversidade e diagnóstico quali-quantitativo. Revista da Sociedade Brasileira de Arborização Urbana, Piracicaba, SP, v. 9, n. 4, p. 80-98, 2014.

PAIVA, A.V. ASPECTOS DA ARBORIZAÇÃO URBANA DO CENTRO DE COSMÓPOLIS -SP. Revista da Sociedade Brasileira de Arborização Urbana, Piracicaba, SP, v.4, n.4, p.17-31, 2009.

PARRY, M.M.; SILVA, M.M.; SENA, I.S.; OLIVEIRA, F.P.N. Composição florística da arborização da cidade de Altamira, Pará. Revista da Sociedade Brasileira de Arborização Urbana, Piracicaba, SP, v. 7, n.1, p. 143-158, 2012.

PIRES, N.A.M.T.; MELO, M.S.; OLIVEIRA, D.E.; XAVIER-SANTO, S. A ARBORIZAÇÃO URBANA DO MUNICÍPIO DE GOIANDIRA/GO - CARACTERIZAÇÃO QUALI-QUANTITATIVA E PROPOSTAS DE MANEJO. Revista da Sociedade Brasileira de Arborização Urbana, Piracicaba, SP, v.5, n.3, p. 185-205, 2010. 
RAUPP, M. J.; CUMMING, A. B.; RAUPP, E. C. Street tree diversity in eastern North America and its potential for tree loss to exotic borers. Arboriculture \& Urban Forestry, Champaign, v.32, n.6, p.297-304, 2006.

SAMPAIO, A.C.F.; DE ANGELIS, B.L.D. INVENTÁRIO E ANÁLISE DA ARBORIZAÇÃO DE VIAS PÚBLICAS DE MARINGÁ-PR. Revista da Sociedade Brasileira de Arborização Urbana, Piracicaba, SP, v. 3, n.1, p. 37-57, 2008.

SANTAMOUR JÚNIOR, F.S. Trees for urban planting: diversity unifomuty, and common sense. Washington: U.S. National Arboretum, Agriculture Research Service, 2002.

SANTOS, C.Z.A.; FERREIRA, R.A.; SANTOS, L.R.; SANTOS, L.I.; GOMES, S.H.; GRAÇA, D.A.S. Análise qualitativa da arborização urbana de 25 vias públicas da cidade de Aracaju-SE. Ciência Florestal, Santa Maria, RS, v.25, n. 3, p. 751-763, jul-set, 2015.

SCHALLENBERGER; L.S.; MACHADO, G.O. INVENTÁRIO DA ARBORIZAÇÃO NA REGIÃO CENTRAL DO MUNICÍPIO DE MANGUEIRINHA - PR. Revista da Sociedade Brasileira de Arborização Urbana, Piracicaba, SP, v.8, n.1, p.54-64, 2013. 